\title{
Ultrafast Fiber Laser Platform for Advanced Materials Processing
}

\author{
Michael Mielke, David Gaudiosi, Kyungbum Kim, Michael Greenberg, Xinhua Gu, Robert Cline, Xiang Peng, \\ Mark Slovick, Neill Allen, Michael Manning, Michael Ferrel, Nath Prachayaamorn, and Steve Sapers
}

Raydiance, Inc., 2199 South McDowell Blvd., Suite 140, Petaluma, CA 94954, USA,

Email: mmielke@raydiance-inc.com

\begin{abstract}
Ultrafast lasers based on fiber optic architecture and integrated software controls will play a dominant role in the spread of these lasers into commercial and industrial applications. The principle challenges are to generate adequate pulse energy from singlemode fiber amplifiers which have small cross-sectional area, and to perform all tuning and control processes with internal electronics and robust software. We demonstrate an all-fiber erbium amplifier system that produces $>100 \mu \mathrm{J}$ per pulse with femtosecond class pulsewidth, excellent beam quality, and an autonomous control system. We present details regarding our laser platform performance and discuss the salient reliability and lifetime topics that are necessary for ultrafast lasers to spread into realistic industrial environments. As examples, we show machining of Nitinol, Teflon, and a biodegradable polymer stent material with no observable heat affected zone. Basic process parameters are described. DOI: $10.2961 / \mathrm{j} 1 \mathrm{mn} .2010 .01 .0012$
\end{abstract}

Keywords: Ultrafast, ultrashort pulse, femtosecond, fiber laser, non-thermal, micro-machining, smart light

\section{Introduction}

Ultrafast lasers have become essential tools for advanced micro-machining and materials processing. When delivered properly to the target, the extreme brevity of the pulses facilitates material removal without imposing thermal effects on the remaining structure. We describe herein a new ultrafast laser platform (Smart Light ${ }^{\mathrm{TM}}$ ) based on fiber-optic laser architecture that is fully integrated with embedded electronics and software layers for autonomous and safe system operation. We show micro-machining examples, technology platform overview, laser system performance data, and preliminary reliability characterization.

\subsection{Non-thermal Material Removal}

There has been considerable dialogue in the laser materials processing community regarding the enabling pulse duration for achieving non-thermal ablation - where the remaining material shows no heat affected zone (HAZ) [1]. The specific solution is process dependent since the laser repetition rate as well as the material feed rate influence spatial heat build-up. Nonetheless, there are general trends that have been well documented, including a pulsewidth requirement based upon the physical optical properties of a given material. The pulsewidth regime for non-thermal ablation is defined by:

$$
\tau_{p}<<\frac{\delta^{2}}{D}
$$

where $\tau_{p}$ is the laser pulse duration full width at half maximum (FWHM) power, $\delta$ is the optical skin depth, and $D$ is the thermal diffusivity of the material [2]. There is some variety in the pulsewidth requirements for different materials since the electron-phonon coupling time can range from a few picoseconds to several tens of picoseconds [3]. Still, an ultrafast laser source that is broadly enabling for nonthermal processing of most materials families should have sub-picosecond FWHM and a tightly confined temporal energy distribution.

\subsection{Nitinol Machining Example}

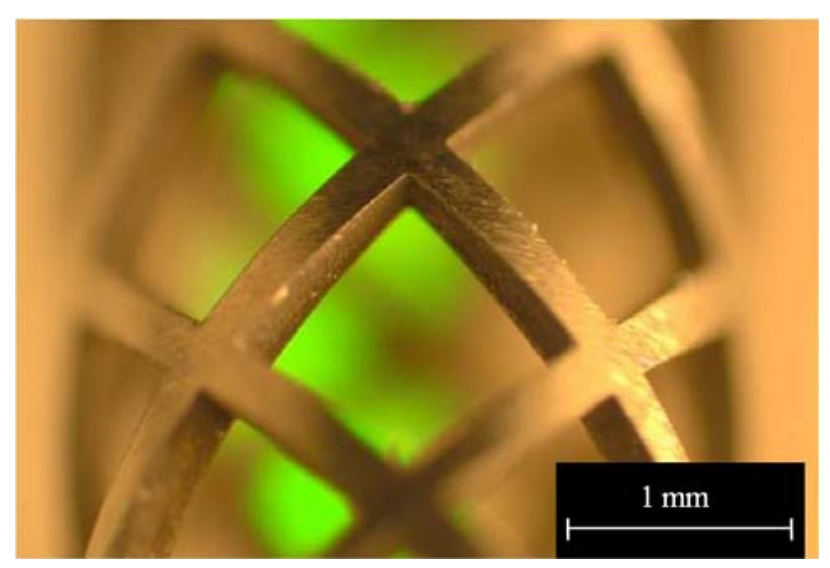

Fig. 1 Visible light microscope image of a nitinol stent machined with the $50 \mu \mathrm{J}$ laser.

A high value, near term commercial application for ultrafast lasers is for manufacture of implantable medical devices, such as vascular stents. The current generation of devices typically comprises metal alloys including Nitinol, a nickel-titanium shape memory alloy. These devices have historically been machined with nanosecond pulse lasers, and several stages of post-processing are needed to remove burrs, slag, and recast [4]. In contrast, Fig. 1 shows a Nitinol stent machined with a Raydiance $50 \mu \mathrm{J}$ laser workstation, where the typical post-processing steps have been avoided owing to the non-thermal material removal enabled by the laser. To be clear, there has been no honing, deburring, or chemical etching performed to remove defects. The laser settings for Nitinol machining are $40 \mu \mathrm{J}$ pulse energy on target, $100 \mathrm{kHz}$ repetition rate, and $800 \mathrm{fs}$ 
pulse duration. The $3 \mathrm{~mm}$ outer diameter (OD), $254 \mu \mathrm{m}$ wall thickness raw tubing is manipulated in a laser lathe style workstation. With $25 \mu \mathrm{m}$ kerf width, the linear machining speed is approximately $1 \mathrm{~mm} / \mathrm{s}$.

Closer inspection of the stent machined edge region provides proof of the non-thermal nature of the machining work performed with our system. Fig. 2 displays a scanning electron microscope (SEM) image with a 500x magnification. The Nitinol stent has been cross-sectioned, and the grain relieved via acid etch. The image shows uniform grain structure from the bulk of the material (far left) straight through to the visible boundary with the mounting epoxy (epoxy is the dark area on the right). Since HAZ is easily revealed by change to the grain structure, this sample is shown to be free of HAZ.

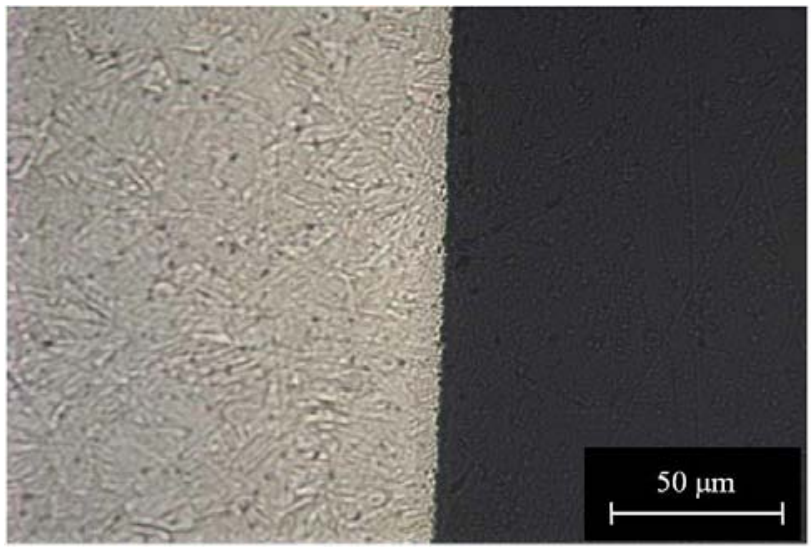

Fig. 2 Scanning electron microscope (SEM) image showing a 500x magnified view of the laser machined Nitinol edge. The uniform grain structure indicates lack of heat affected zone (HAZ).

The key advantages of non-thermal processing of Nitinol stents are the ability to dramatically reduce the material thickness, to reduce the lattice cross bar or strut width, and to greatly improve the device yield since the bypassed postprocessing steps often cause catastrophic damage. The ultrafast platform moreover enables laser machining of entirely new classes of materials and devices which simply could not be machined in a thermal manner. These include certain hard dielectrics, plastics, and polymers infused with medicines.

\subsection{Polymer Machining Example}

We have utilized the Raydiance $50 \mu \mathrm{J}$ lasers for precision drilling of holes in soft, heat sensitive materials as well as for creating prototype stents made of biodegradable polymers that dissolve inside the human body after a prescribed treatment period. Fig. 3 shows an example of drilling an $830 \mu \mathrm{m}$ diameter hole through the sidewall of a Teflon tube. The picture shows the machined area directly after laser drilling without any clean-up of debris, hence there is some particulate matter held onto the substrate by static cling. There is no evidence of melt or discoloration. The particulate matter that has collected on the sample was easily removed by wiping with isopropyl alcohol (IPA). The laser settings for Teflon machining are $30 \mu \mathrm{J}$ pulse energy on target, $100 \mathrm{kHz}$ repetition rate, and $800 \mathrm{fs}$ pulse duration. We used a galvanometer style laser scanner to drill the $830 \mu \mathrm{m}$ hole through $380 \mu \mathrm{m}$ thick material in approximately 5 seconds. Hence the volumetric material removal rate is $0.041 \mathrm{~mm}^{3} / \mathrm{s}$.

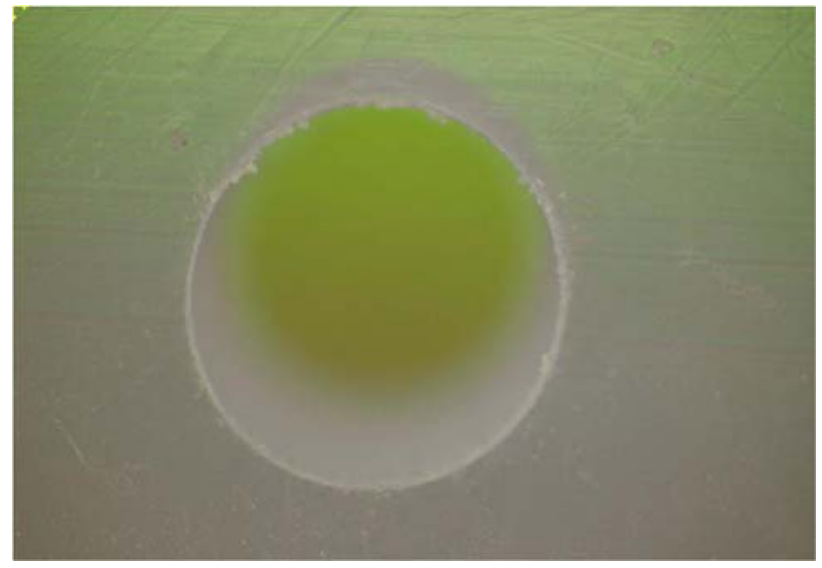

Fig. $3830 \mu \mathrm{m}$ diameter hole drilled through polytetrafluoroethylene (Teflon) with the $50 \mu \mathrm{J}$ laser.

We have fabricated several types of implantable medical devices from biodegradable polymer substrates. Fig. 4 shows the edge view of a polymer tube that has been cut using a Raydiance $50 \mu \mathrm{J}$ laser. Similar to the Teflon picture, there is no evidence of melt or discoloration. There is merely a small volume of debris that can be removed with IPA wipe or brief ultrasonic bath. The laser settings for biodegradable polymer machining are $44 \mu \mathrm{J}$ pulse energy on target, $33 \mathrm{kHz}$ repetition rate, and $800 \mathrm{fs}$ pulse duration. The $150 \mu \mathrm{m}$ thick polymer tubing is cut with a linear machining speed of approximately $1.4 \mathrm{~mm} / \mathrm{s}$.

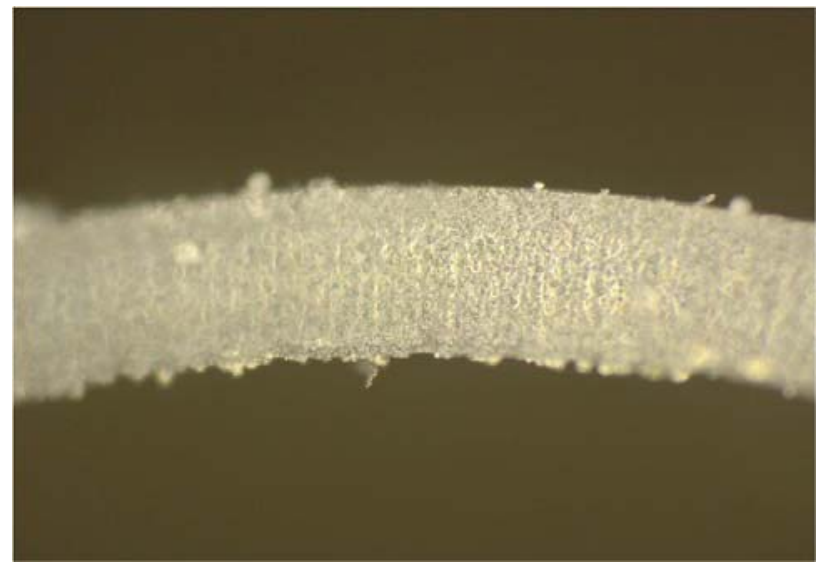

Fig. 4 Edge view of a biodegradable polymer stent showing a cut made with the $50 \mu \mathrm{J}$ laser.

The superior laser processing capability illustrated in these examples has been consistent for all materials we have attempted to machine. Furthermore, most of the processes require less than one workday to demonstrate since the laser parameters are quickly adjusted to downselect to the optimum configuration. Next we describe the platform architecture that provides this ease-of-use and rapid process execution. 


\section{Laser Platform Architecture}

We created a fiber-optic chirped pulse amplification (CPA) architecture at $1.55 \mu \mathrm{m}$ wavelength as a basis for our ultrafast laser platform. We merged this with sophisticated analog and digital electronics and a robust software operating system in the initial stage of development, and the functional layers have evolved in synchronicity over the past several years. As described below, this allowed immediate reliability and autonomous system management that were not previously demonstrated by titanium sapphire or other types of ultrafast laser architectures. As we have expanded the pulse energy handling of the fiber-optic amplifiers, the list of practical applications for the platform has grown quickly.

\subsection{Fiber-Optic CPA Design}

Despite the challenges associated with generating very high peak irradiance pulses from fiber amplifiers, the scalability of the architecture is inherent in this framework. Throughout the 1990s, there occurred a massive global investment in optical telecommunications at the $1.55 \mu \mathrm{m}$ wavelength. Many optical components were commoditized, and very high quality passive and active fiber types became standard catalog options. Test and quality standards, such as Telcordia specifications, helped improve the lifetime and performance determinism of fiber-optic systems [5]. Though we use several specialized subassemblies, we have overall leveraged the global investment to great benefit for ultrafast laser materials processing systems. In addition, volume production, cost reduction, and enhanced consistency are native aspects of the platform.

Fig. 5 shows a sketch of the Raydiance fiber-optic CPA architecture. The mode-locked laser, the pulse stretcher, the optical amplifiers, and all the intermediate components (isolators, taps, etc.) are truly fiber-optic units. They are assembled using fiber fusion splicers operated by trained technicians, and these laser systems are manufactured in less than two weeks, start-to-finish. The pulse compressor still comprises discrete, free-space optics. Yet our ruggedized opto-mechanical design permits product shipment by standard carrier and system installation in less than thirty minutes. Further technical detail of the laser subsystem performance has been described previously [6]. The overall laser output is described below in sections 3 and 4 .

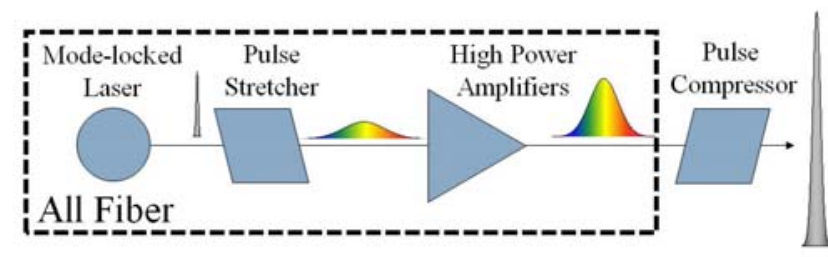

Fig. 5 Ultrafast laser optical scheme based on chirped pulse amplification (CPA). Raydiance lasers employ all-fiber subassemblies except for the final pulse compressor.

\subsection{Operating System}

The optical layer is integrated with our own dedicated printed circuit boards (PCBs) that incorporate the latest generation microprocessors as well as an industry standard single board computer (SBC). The SBC runs an embedded software control system that manages the laser operations on multiple levels. A Linux kernel directly manages the low level device drivers, e.g. laser diode controllers, analog-to-digital (ADC) interfaces, etc. An intermediate layer directs high level subassembly instructions, pulls settings from EEPROM registers, and exports laser performance information to our logging system. At the top level, our applications programming interface (API) accepts commands from the laser user, translates those commands to the operating system, and ensures the instructions are consistent with safe laser operation.

Raydiance laser systems are shipped with a notebook computer that runs our graphical user interface (GUI) that is displayed in Fig. 6. The GUI executable program connects to the laser system via standard intranet service and provides instructions to the laser via the API. The GUI provides user control of the pulse repetition rate, the pulse energy, the average power, pulse packet mode programming, triggering functions, and a visible aiming beam. These features are controlled by a mouse click as opposed to the conventional solid state laser paradigm where manual hardware change has been essential.

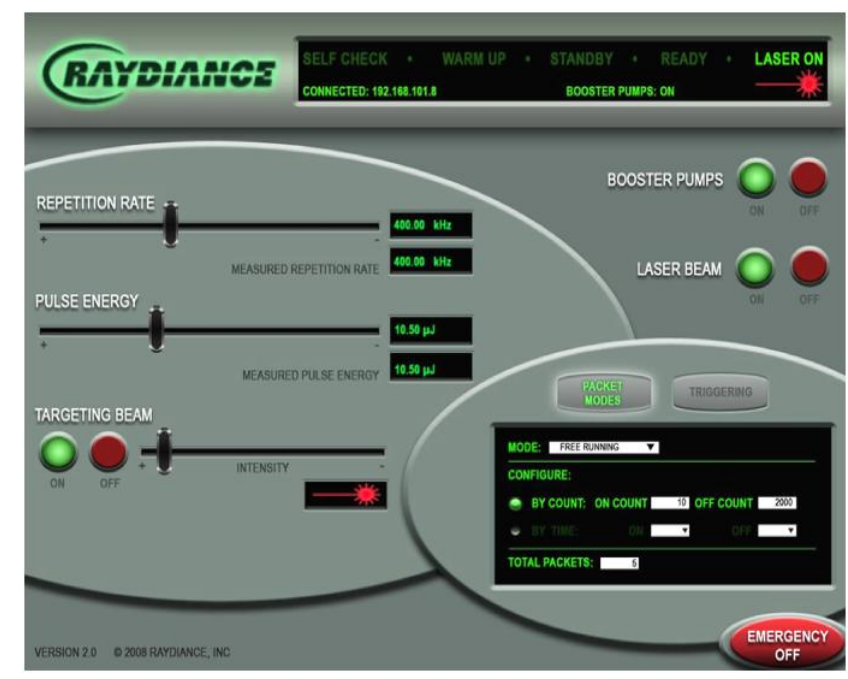

Fig. 6 Graphical user interface (GUI) provided with Raydiance lasers. Pulse energy, repetition rate, packet mode programming, and visible aiming beam are each independently controlled via computer mouse click.

The embedded software operating system sustains stable and consistent laser operation. The Raydiance laser systems are in service 24 hours per day, seven days per week with no maintenance or tuning required. The GUI gives the user immediate access to a diverse range of optical performance points for ready process development. The superior performance of this ultrafast laser system design is described next.

\section{Basic Performance Data}

In application of ultrafast laser light, the process success usually depends upon the confinement of the laser energy in both time and spatial domains. Here we describe the laser system temporal pulse quality at two different operating points, plus we demonstrate excellent spatial beam quality using the traditional beam propagation ratio, or $\mathrm{M}^{2}$, plus a more thorough analysis technique. 


\subsection{Time Domain Pulse Quality}

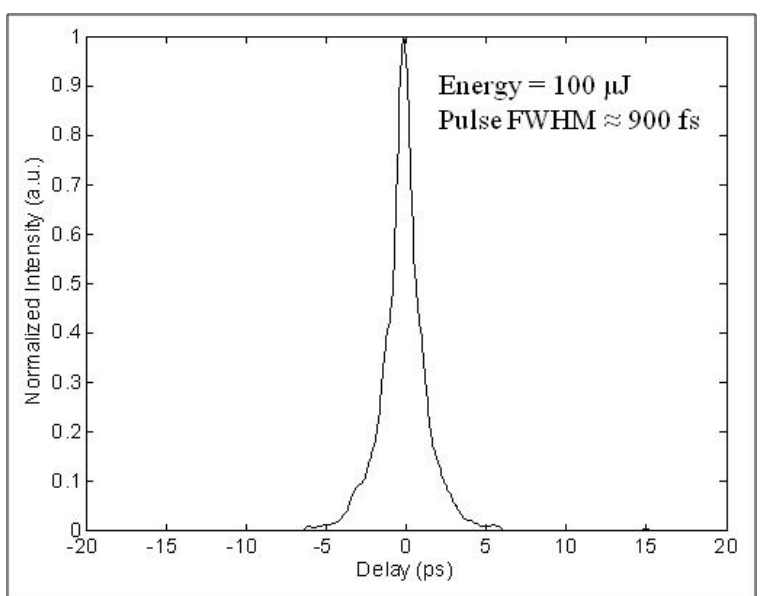

Fig. 7 Typical pulse intensity autocorrelation for the Raydiance platform when operating at $100 \mu \mathrm{J}$ per pulse energy level. Deconvolved pulsewidth is approximately $900 \mathrm{fs}$.

The most widely used temporal pulse measurement method is the optical pulse intensity autocorrelation. It is relatively simple to perform the measurement and data is captured using a modest bandwidth voltage oscilloscope. Fig. 7 shows the intensity autocorrelation for one of our Smart Light ${ }^{\mathrm{TM}}$ laser systems when operating at $100 \mu \mathrm{J}$ pulse energy and $3.5 \mathrm{~W}$ average power $(35 \mathrm{kHz}$ pulse repetition rate). Similarly, Fig. 8 shows the intensity autocorrelation for the same Smart Light ${ }^{\mathrm{TM}}$ laser system when operating at $50 \mu \mathrm{J}$ pulse energy and $5.0 \mathrm{~W}$ average power (100 $\mathrm{kHz}$ pulse repetition rate).

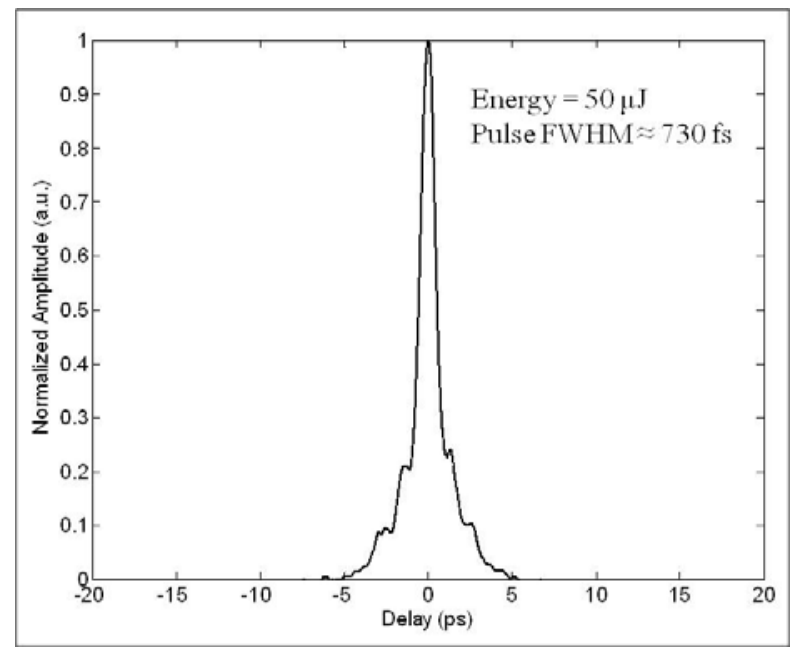

Fig. 8 Typical pulse intensity autocorrelation for the Raydiance platform when operating at $50 \mu \mathrm{J}$ per pulse energy level. Deconvolved pulsewidth is approximately $730 \mathrm{fs}$.

There is a small amount of pulse broadening (to $900 \mathrm{fs}$ ) when the system was configured for $100 \mu \mathrm{J}$ versus operation at $50 \mu \mathrm{J}$ where the FWHM was $730 \mathrm{fs}$. This broadening is due to modest self-phase modulation (SPM) accumulated in the final fiber amplifier stage. In both cases, nonetheless, the pulse energy is well-confined within the $10 \mathrm{ps}$ centroid centered at the pulse peak. This is a key performance parameter for ultrafast lasers since broad temporal pedestals can cause heat build-up in the machined material thereby negating the benefit of the sub-picosecond pulse FWHM. Raydiance Smart Light ${ }^{\mathrm{TM}}$ laser systems are specified to have sub-picosecond pulse FWHM and to have at least $90 \%$ of their energy within the 10 ps pulse centroid.

\subsection{Spatial Beam Quality}

The beam quality figures-of-merit are critical to understanding the capability for focusing or collimating and propagating a laser beam with determinism. Beam quality is usually quantified with the beam propagation ratio, or $\mathrm{M}^{2}$, and the measurement method is well-documented (ISO 11146-2), although this protocol is not always strictly followed by laser manufacturers. Moreover, it is a rather coarse description of beam quality that reveals only the departure from an ideal Gaussian beam. For these reasons, we supplement the $\mathrm{M}^{2}$ measurement with a recently developed technique called the spatially and spectrally resolved imaging method, or $\mathrm{S}^{2}$ [7].

With the $S^{2}$ method, the near field beam profile from the laser is sampled with good resolution (a few microns) and the optical spectrum is recorded for each pixel. A simple Fourier transform algorithm is then used to extract the modal content of the beam. As shown in Fig. 9, the fundamental mode (FM) is often accompanied by at least some power in higher order fiber modes. The relative magnitude of power in the higher order modes can reveal flaws in fiber splicing, mode matching offset, or mechanical strain on lower numerical aperture fibers. In our systems, we typically observe higher order mode suppression of better than $20 \mathrm{~dB}$, hence the composite beam appears to contain only the FM. The corresponding beam propagation ratio for this laser is approximately $\mathrm{M}^{2}=1.3$ as measured with a commercial scanning knife-edge beam profiler.

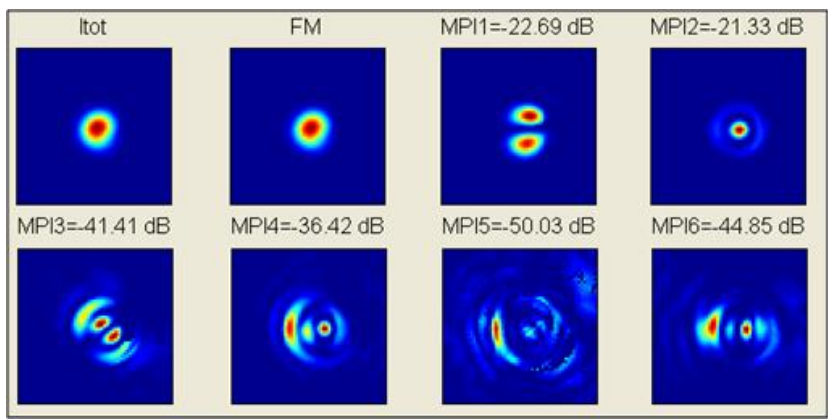

Fig. 9 Spatial mode analysis of a typical large mode area (LMA) erbium fiber amplifier used in the Raydiance platform. The data extracted from an $\mathrm{S}^{2}$ (S-squared) measurement reveals at least $20 \mathrm{~dB}$ suppression of multi-path interference (MPI) from higher order modes relative to the fundamental mode (FM) intensity.

The basic laser performance data shown here demonstrates the Raydiance lasers can perform non-thermal machining with well-defined beam focus and spot size. These are necessary, though not sufficient, conditions for supporting the emerging industry using ultrafast lasers. Ultrafast laser reliability has been notoriously awful - with the lasers requiring frequent hands-on attention from specially trained laser engineers. In the next section, we prove that this ultrafast laser paradigm is no longer the only option. Rather, 
Raydiance lasers operate continuously around the clock without user tuning or down-time.

\section{Preliminary Reliability Data}

In this final section of laser performance description, we display two key characteristics of long term laser operation and stability: pulse quality versus time and temperature; and output average power versus time and temperature. In the data sets shown here, the lasers are running continuously on our manufacturing floor where the room temperature is regulated only during normal business hours. Hence the slow rise in temperature indicates the cooling and ventilation system is disengaged. We believe these long term observations with variable ambient temperature are crucial to engineering and providing lasers that function properly in realistic manufacturing scenarios. We make similar observations of each laser we manufacture during the system burn-in period.

Fig. 10 shows two pulse temporal quality figures-ofmerit for one of our $50 \mu \mathrm{J}, 5 \mathrm{~W}$ systems: the temporal FWHM (top) and the integrated energy within the 5 ps centroid (middle) centered at the pulse peak. Note this is a stricter pulse energy confinement test than mentioned previously. The bottom chart shows the ambient temperature for the manufacturing floor measured several feet from the laser system. The FWHM is quite stable near $800 \mathrm{fs}$ for the duration of the test ( 3.75 days, continuous), and the integrated energy in 5 ps never drops below $90 \%$, despite the ambient temperature fluctuating between 23 and $29^{\circ} \mathrm{C}$.

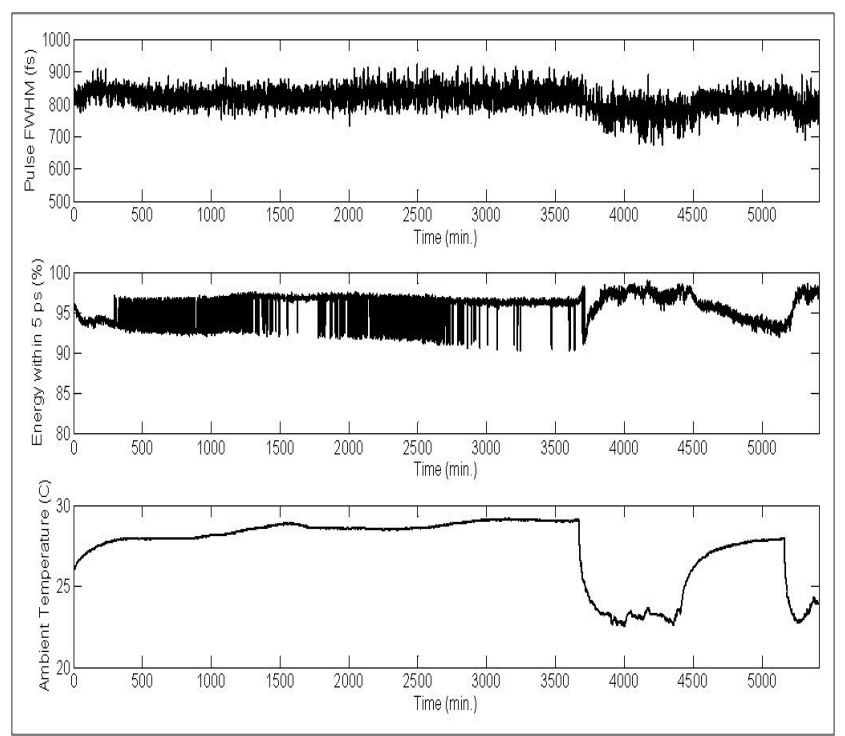

Fig. $1050 \mu \mathrm{J}, 5 \mathrm{~W}$ system output pulse duration FWHM (top) and percentage of pulse energy within the 5 ps pulse centroid (middle) versus ambient temperature (bottom) over a period of 3.75 days. These stability levels are representative of the product line.

We similarly measured the average power output from a $50 \mu \mathrm{J}, 5 \mathrm{~W}$ laser system over time and temperature, and the data is shown in Fig. 11. The measured power remains flat for the duration of the observations (50 hours), though the ambient temperature rises from 24 to nearly $29{ }^{\circ} \mathrm{C}$. We follow the ISO 11554 prescribed power fluctuation measurement protocol, $\Delta P=2 \sigma / P_{\text {mean }}$, where $\sigma$ is the standard deviation, and $\Delta P<1 \%$ for the data set.

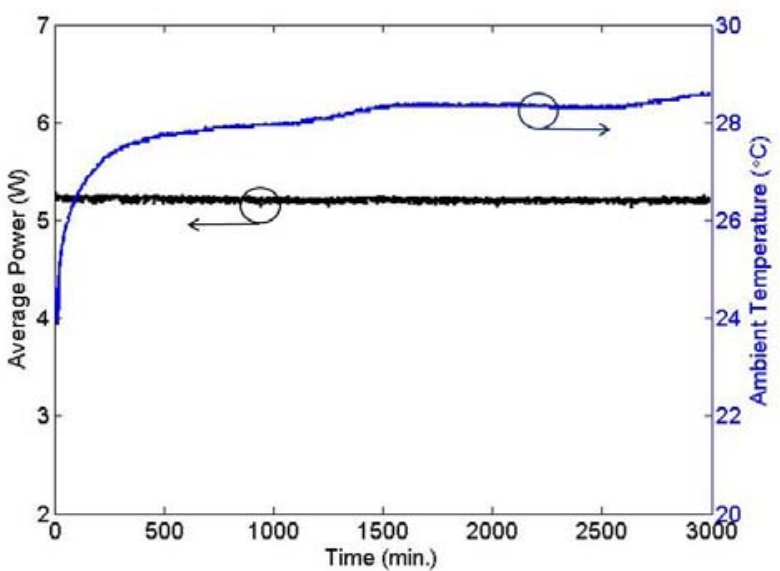

Fig. 11 Laser system average power and the ambient temperature for approximately 50 hours of continuous measurement time. Power fluctuation is less than $1 \%$ (following ISO 11554 measurement protocol).

These stability figures may not seem remarkable when compared to many standard types of industrial equipment. When compared to other ultrafast lasers, however, these figures are extraordinary and represent a paradigm shift for this broadly enabling platform tool. We have also conducted preliminary laser lifetime tests for a previous version of the Smart Light ${ }^{\mathrm{TM}}$ product line $(10 \mu \mathrm{J}, 3 \mathrm{~W}$ maximum output). The tests indicate approximately 27,000 hours will elapse before the first service intervention is required. There are several major subassembly changes between the versions, yet we expect similar lifetime performance for the 50 and $100 \mu \mathrm{J}$ versions.

\section{Summary}

Many of us expect the next century to be revolutionized by photonics in a manner similar to how the last century was changed by micro-electronics and micro-processors. The first enabling technologies are presently branching out from the photonics laboratory and into commercial and industrial arenas. We have illustrated critical performance data for Raydiance ultrafast lasers, plus glimpses of some early applications examples for this unique and powerful form of light. The Smart Light ${ }^{\mathrm{TM}}$ platform is ready to drive high value micro-machining applications.

\section{References}

[1] For example: P.S.Banks, et al., "Femtosecond Laser Materials Processing," Proc. SPIE 3934, pp. 14-21, 2000.

[2] For example: S.S.Wellershoff, et al., "The role of electron-phonon coupling in femtosecond laser damage of metals," Appl. Phys. A 69 [Suppl.], S99-S107 (1999).

[3] For example: F.Korte, et al., "Far-field and near-field material processing with femtosecond laser pulses," Appl. Phys. A 69 [Suppl.], S7-S11 (1999).

[4] For example: B.N.Chichkov, et al., "Femtosecond, picosecond and nanosecond laser ablation of solids," Appl. Phys. A 63, 109-115 (1996).

[5] For example: Telcordia GR-20, "Generic Requirements for Optical Fiber and Optical Fiber Cable," 2008. 
[6] M.Mielke, et al., "Pulse and Amplifier Dynamics in High Energy Fiber Optic Ultrashort Pulse Laser Systems," Proc. SPIE 7214 (7214-30) 2009.
[7] J.W.Nicholson, et al., "Spatially and spectrally resolved imaging of modal content in large-mode-area fibers," Opt. Express 16, pp.7233 (2008). 